\title{
Effect of feature similarity on illusory conjunctions
}

\author{
RICHARD B. IVRY and WILLIAM PRINZMETAL \\ University of California, Santa Barbara, California
}

\begin{abstract}
In four experiments, we examined whether the phenomenon of illusory conjunctions is constrained by feature similarity. Specifically, are illusory conjunctions more likely to occur between items with similar features than between items with dissimilar features? Feature similarity was manipulated in two dimensions: color and shape. Experiment 1 demonstrated that more illusory conjunctions occur between items with similar colors than between items with dissimilar colors. A similar effect was found for letter similarity in Experiment 2. Experiments 3 and 4 demonstrated that the similarity effect is still obtained for illusory conjunctions even when identification of the relevant features is near perfect. These findings introduce a new constraint in the process of feature integration. Additional implications of the similarity constraint for theories of feature integration are discussed.
\end{abstract}

When multidimensional stimuli are presented briefly, subjects can perceive the component features in incorrect combinations (Treisman \& Schmidt, 1982). For example', if a display contains a red $O$ and a blue $X$, subjects will occasionally perceive a blue $O$. These incorrect combinations, referred to as illusory conjunctions, provide a direct method for investigating processes involved in feature integration. Stimulus manipulations that influence the likelihood of illusory conjunctions can be used to indicate how the visual system combines features. In this paper, we investigate whether color and shape similarity affect the formation of illusory conjunctions.

Five variables have been shown to constrain the formation of illusory conjunctions. First, it has been found in several studies that illusory conjunctions are more likely to occur between items that are adjacent in a display in comparison with items that are distant (Cohen \& Ivry, 1989; Gallant \& Garner, 1988; Keele, Cohen, Ivry, Liotti, \& Yee, 1988; Wolford \& Shum, 1980).

Second, illusory conjunctions are affected by the spread of attention. Cohen and Ivry (1989) manipulated the spread of attention with a primary task in which subjects identified two neutrally colored digits. The colored letters for the conjunction task were at fixed locations, but they were sometimes between the digits (within the spread of attention) and sometimes outside the digits (outside the spread of attention). Cohen and Ivry (1989) found that illusory conjunctions were more likely to occur when both items were within the spread of attention. Moreover,

\footnotetext{
This work was supported by Office of Naval Research Contract N00014-87-K-0279 to S. Keele and R. Ivry and by NIMH Grant MH39881 to W. Prinzmetal. The authors are grateful to Asher Cohen, Greg Ashby, Anne Treisman, Ray Klein, Jeremy Wolfe, and Lester Krueger for their comments and discussions regarding this research. Reprint requests should be sent to R. Ivry, Department of Psychology, University of California, Berkeley, CA 94720.
}

manipulation of the distance between two colored letters that lay within two fixed digit locations had a small effect on the probability of illusory conjunctions. Treisman and Schmidt (1982) had failed to find a distance effect in a similar paradigm, in which digits were used to ensure that attention was spread over all of the items in a display. Generally, illusory conjunctions are inversely related to distance, but this effect is diminished when the candidate features are presented within the spread of attention.

Third, perceptual organization can also modify the effect of distance between items (see, e.g., Prinzmetal, 1981; Prinzmetal \& Keysar, 1989). Prinzmetal (1981) manipulated perceptual grouping with good continuation or similarity while controlling for the distance between features. Illusory conjunctions between features within the same perceptual group were more likely than conjunctions between features in different perceptual groups.

Fourth, feature integration can be affected by grouping determined by purely cognitive factors. Prinzmetal and Keysar (1989) found that subjective organization has the same effect as proximity grouping. Ilusory conjunctions are also constrained by linguistic factors. Prinzmetal, Treiman, and Rho (1986) discovered that illusory conjunctions of colors were less likely between letters in different syllables than between letters within a syllable in words and pseudowords (also see Prinzmetal \& MillisWright, 1984; Prinzmetal, 1990; Seidenberg, 1987).

Finally, temporal proximity can affect illusory conjunctions: features that successively appear far apart in time at the same location are less likely to be joined than features that appear close in time (McLean, Broadbent, \& Broadbent, 1982). However, when Keele et al. (1988) pitted spatial proximity against temporal proximity, illusory conjunctions were more likely to occur between features at the same location that were not temporally adjacent, rather than temporally contiguous. 
In summary, the visual system avoids joining features that are far apart in space or time, are segregated by attention, or are from different perceptual or subjective groups. In general, these constraints would prevent the visual system from wrongly combining features from different objects in the real world.

In the current experiments, we attempted to examine whether feature similarity might also constrain feature integration. If this were so, we would expect that subjects would be more likely to incorrectly report that the color of a target letter was the color of another display letter if the two letters were similar in color (e.g., green and blue) than if they were dissimilar in color (e.g., green and red). Analogously, similarity effects may be found in letter space. Subjects may be more likely to incorrectly report the letter of a target color if the display letters are similar in shape (e.g., T and L) than if they are dissimilar in shape (e.g., $T$ and $W$ ). The use of psychological scaling techniques (e.g., those of Shepard, 1980) has demonstrated that the relative similarity between perceptual features such as color and form can be conceived as the distance between stimuli in a multidimensional space. Just as illusory conjunctions are more likely to occur between items that are spatially close to each other, they may be more likely between items in feature space.

However, there are theoretical and empirical reasons for predicting that illusory conjunctions would not be influenced by feature similarity. As discussed previously, the occurrence of illusory conjunctions is affected by the spatial distance between features. One possible mechanism underlying illusory conjunctions may be that location information is poorly registered during the identification of features. This might occur because the location information is crude (Cohen \& Ivry, 1989; Prinzmetal \& Keysar, 1989) or because such information is subject to rapid decay (Wolford, 1975). For example, if the color of one item and the shape of a different item were perceived as sharing a common location, they would be reported as belonging to a single object. The issue then becomes whether location and feature information are determined by the same mechanism or different mechanisms. Many models of visual perception assume that location and featural information are processed independently in terms of both cognitive operations and anatomical pathways (e.g., Levine, Warach, \& Farah, 1985; Newcombe, Ratcliffe, \& Damasio, 1987; Treisman \& Gelade, 1980; Ungerleider \& Mishkin, 1982). According to these models, there is no reason to suppose that location information is worse for displays containing similar features than it is for displays containing dissimilar features.

On the other hand, illusory conjunctions may be due to poor location information, but location and feature information may be processed by the same mechanism. In this case, both location and feature similarity might affect illusory conjunctions. There are at least two reasons for this prediction. First, similar items may form perceptual groups, leading to more illusory conjunctions (Prinzmetal, 1981; Prinzmetal \& Keysar, 1989). Second,
Prinzmetal and Keysar (1989) performed a computer simulation in which it was assumed that illusory conjunctions were due to poor spatial location for color (i.e., color spreading). In the simulation, they found that a target's color was influenced more when the target was surrounded by similarly colored noise than when it was surrounded by dissimilar noise. Similar colors tended to combine more readily than dissimilar colors.

Only Treisman and Schmidt (1982, Experiment 4) have tested whether or not illusory conjunctions are affected by feature similarity. They found no difference in the proportion of illusory combinations of color between items with identical shape, size, and solidity (i.e., filled-in or outlined), in comparison with items that differed on these dimensions-a result which indicates that illusory conjunctions are not affected by feature similarity. However, the question of whether or not similarity can influence feature integration should not be decided by one null result. Furthermore, Treisman and Schmidt (1982) varied similarity between dimensions. They found, for example, that objects identical in shape were no more likely to produce illusory conjunctions between two other dimensions (color and solidity) than objects different in shape. Yet it is possible that similarity within a dimension (e.g., color, shape) would affect illusory conjunctions involving that dimension. Hence, in the following experiments, we tested whether feature similarity of color (Experiments 1 and 3 ) or shape (Experiments 2 and 4) would affect illusory conjunctions.

Although the question of whether feature similarity affects illusory conjunctions is in principle straightforward, we faced an insidious methodological problem (also see Prinzmetal, Presti, \& Posner, 1986). Consider two displays: one contains a red $\mathrm{X}$ and a blue $\mathrm{O}$, the other a green $X$ and a blue $O$. If illusory conjunctions are more likely to occur with similar colors, then a subject should be more likely to erroneously report "blue $X$ " following the second display, since green and blue are more similar than red and blue. However, such a result would not necessarily mean that similarity affected illusory conjunctions. A briefly presented green letter will be more likely to be reported as blue than as red, regardless of whether or not there are other colors in the display. In Treisman's terminology (Treisman \& Schmidt, 1982), a failure to correctly perceive a feature is labelled a feature error, and these errors can be expected to show the effects of similarity. Thus, in the example above, the illusory blue $\mathrm{X}$ may indicate that the subject misperceived the feature (feature error) or that the subject incorrectly combined correctly perceived features (conjunction error). Our methods and analyses were designed to provide converging approaches to overcoming this problem.

\section{EXPERIMENT 1}

We based Experiment 1 on the method of Cohen and Ivry (1989). Each trial began with the presentation of an alerting signal that consisted of two horizontal bars, one 
located to the left and one to the right of the center of the display. The bars were then replaced with a briefly presented multielement display that contained two achromatic digits and two colored letters. The digits were located just inside the bars and were included to ensure that the subjects spread their attention over the entire region between the two positions. The subjects were instructed that the primary task was to accurately determine whether the digits were the same. The colored letters were positioned in one of four locations between the two digits. One letter, the target, was an X or a T, and the other, the distractor, was an $O$ or an $S$. The subject reported the color of the $\mathrm{X}$ or $\mathrm{T}$ and indicated whether the target letter was an $\mathrm{X}$ or a $\mathrm{T}$.

The critical manipulation in Experiment 1 involved the degree of similarity between the target color and the distractor color. Four colors were used: red, orange, green, and blue. Two pairs of the colors are similar (red and orange; blue and green) whereas the other combinations (e.g., red and green) are dissimilar. If illusory conjunctions are affected by feature similarity, we expected that there would be more illusory conjunctions when the target and distractor colors were similar than when they were dissimilar.

\section{Method}

Subjects. Twelve students were recruited from the undergraduate subject pool at the University of California, Santa Barbara. The subjects received course credit for their participation. All of the subjects had normal or corrected-to-normal vision and reported normal color vision.

Stimuli. The stimuli were presented on a Zenith color monitor (ZCM-1490, vertical synch rate $=70 \mathrm{~Hz}$ ) controlled by an AST computer (AST Premium 286) equipped with a video graphics adapter (AST-VGA Plus). The stimuli consisted of the set of digits 1-9 and the letter set $X, T, O$, and $S$.

The letters were presented in one of four colors: red, orange, green, and blue. These colors were created by adjusting the red, green, and blue video inputs (6-bit resolution) to produce four colors of approximately equal brightness. They were selected to form four corners of a crude rectangle in psychological color space (Indow, 1988). The shorter sides of the rectangle connected the similar-color pairs, red/orange and green/blue. The longer sides connected two of the dissimilar-color pairs, red/blue and orange/green, whereas the diagonals connected the other dissimilar-color pairs, red/green and orange/blue. Thus, the distances between the similar pairs were approximately equal and considerably less than that between any of the dissimilar pairs. The initial color selection was based on the evaluations of the authors and two independent observers, as well as pilot experimentation. The selected colors were matched by four observers (including one of the authors) to Munsell chips (Munsell Color Company, 1929). The average hue, chroma, and value matches were, respectively: red $(5 R, 8.75,4.5)$; orange (6YR, 9.5, $6.0)$; green (7.5GY, 8.25, 5.75); and blue (5PB, 9.75, 4.75).

Two horizontal bars served as the alerting stimulus. The bars and digits were presented in black. All of the stimuli were presented on a moderate gray background.

Before each trial, two digits, two letters, two colors, and the locations for the colored letters were determined in the following manner. First, a digit was randomly selected from the set 1-9. On approximately half the trials, the second digit was set equal to the first digit. On the remaining trials, the second digit was randomly selected from the set 1-9, excluding the value of the first digit. Sec- ond, two letters were randomly selected with the constraint that one letter be either an $X$ or a $T$ and the other letter be either an $O$ or an S. Third, two colors were determined, one assigned to the target letter $(X$ or $T$ ) and one assigned to the distractor letter $(O$ or $S$ ). The location of the colored letter pair was randomly determined, as was the order of the letters within the letter pair.

The stimulus display for a representative trial is depicted in Figure 1. Subjects were seated approximately $100 \mathrm{~cm}$ from the monitor, and all distances are given in degrees of visual angle. The alphanumeric characters covered an area that spanned 20 pixels per side, subtending visual angles of $0.43^{\circ}$ horizontally and $0.60^{\circ}$ vertically. Each horizontal bar subtended a visual angle of $0.53^{\circ} \times$ $0.12^{\circ}$. The two markers were horizontally displaced so that the outer boundaries spanned a distance of $6.07^{\circ}$. The digits were presented inside the markers and were separated by $4.35^{\circ}$. The pair of colored letters was positioned to either the left or the right of the center of the screen, the location for a given trial being randomly determined. The outermost edge of the colored letter pair was displayed $1.09^{\circ}$ from the center. The horizontal distance between the two letters was $0.11^{\circ}$.

A colored pattern mask was used to terminate the stimulus display. The mask was composed of a grid of small rectangles, each $0.22^{\circ} \times 0.30^{\circ}$, that filled an $8.64^{\circ} \times 1.49^{\circ}$ region. Thus, the mask covered all of the stimuli, including the horizontal bars. The color of each small rectangle was selected from a subset of six default colors available with Turbo Pascal (Borland Version, 5.0), which included blue, green, cyan, red, magenta, and yellow. The arrangement of the grid was invariant across trials, but the color of each small rectangle was randomly determined for each trial.

Procedure. Each trial began with the presentation of the alerting stimulus. One second later, the digits and colored letters were added to the display. After a variable period of time (see below), the stimulus display was replaced by the colored pattern mask for $200 \mathrm{msec}$. The subject then entered three responses by typing designated keys on the computer keyboard. First, they reported whether the digits were the same or different by typing either a " 1 " or a " 0 ," respectively. Second, they reported the color of the target letter by pressing " 1, , " 2 ," " 3 ," or " 4 " for the red, orange, green, or blue, respectively. Third, they reported the identity of the target letter, using " 1 " to indicate " $X$ " and " 2 " for " $T$." Labels were taped above the keys, so that the subjects knew that the response set was limited to the four colors and two letters in the displays. Feedback was only provided after erroneous responses on the digit and letter identity tasks or when the subjects reported a color not included in the display. The feedback consisted of a message, displayed on the monitor for $1 \mathrm{sec}$, that indicated the type of error(s) made. Note that subjects were not given any feedback when they made an illusory conjunction (i.e., reporting the color of the distractor letter). Although this might have introduced a bias favoring illusory conjunctions, the effect would have been the same for trials with similar and dissimilar colors. If the subjects entered

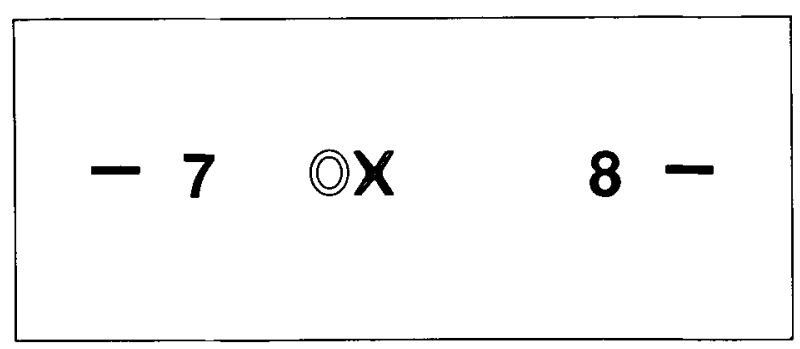

Figure 1. Stimulus display for a representative trial in Experiment 1 . The onset of the fixation markers occurred $1 \mathrm{sec}$ prior to the digits and colored letters. 
more than three responses or entered the information in the incorrect order, all of the data from that trial were eliminated. The intertrial interval was $1 \mathrm{sec}$.

Each subject was tested individually in a sound-attenuating room with overhead fluorescent lighting. After they sat down, the subjects were required to name the four stimulus colors as a simple check of their color vision. The task was then explained as the experimenter stepped through 5 demonstration trials. The subjects were then given four practice blocks of 20 trials each. The exposure duration of the digits and colored letters was reduced for each successive practice block. The exposure durations were 300, 186, 129, and $86 \mathrm{msec}$ for Practice Blocks 1-4, respectively. At the end of each block, the total numbers of correct digit, letter, and color (including illusory conjunctions) responses were displayed, as was the grand total of correct responses.

Following the fourth practice block, the exposure duration for the first experimental block was determined on the basis of the subject's individual performance. If the proportion of correct responses was $95 \%$ or greater, the exposure duration for the first block was reduced by 1 vertical synch cycle to $71 \operatorname{msec}$ (at $70 \mathrm{~Hz}, 1$ cycle $=14.28 \mathrm{msec}$ ). If the percentage was less than $90 \%$, the exposure duration was increased to $100 \mathrm{msec}$; otherwise, the exposure duration remained at $86 \mathrm{msec}$. Using the same criterion, the exposure duration on the second block was either increased or decreased by 1 vertical synch cycle from that of the first block, or remained unchanged. The mean exposure time over all experimental blocks was $91.7 \mathrm{msec}$ (range: $71-100 \mathrm{msec}$ ).

There were three types of trials. The target $(\mathrm{X}$ or $\mathrm{T})$ and the distractor ( $O$ or $S$ ) were identical in color (e.g., both red), similar in color (e.g., green and blue), or dissimilar in color (e.g., red and green). We will refer to the three types of trials as the identical, similar, and dissimilar conditions. Identical trials were included to avoid a response bias based on the distractor color. For example, if there were no identical trials and the subject only perceived the distractor color on a given trial, he or she would be biased against guessing that color as the target color. All possible combinations of color pairs were presented equally often over a block of experimental trials, including identical pairs. Thus, the color of one item provided no information about the color of the other item.

Each subject completed two experimental blocks of 96 trials each. Given that there were 16 color combinations ( 4 target colors $\times 4$ distractor colors), each color condition was presented 6 times per block or 12 times total. The experimental session lasted approximately $1 \mathrm{~h}$.

\section{Results and Discussion}

The digit task was included to ensure that subjects deployed their attention over the entire region within which the stimuli might appear. The subjects were correct on $88.0 \%$ of the trials. This error rate is comparable to that obtained by Cohen and Ivry (1989) on a digitidentification task with a similar display. Most important was that the error rate on the digit task for trials containing dissimilar colors was not significantly different than that for trials with similar or identical colors $[F(2,22)=$ $1.28, p>0.25$ ]. Performance on the colored letter task was unaffected by whether the digits were correctly compared. Thus, the analyses reported below of the colored letter task include all trials, regardless of performance on the digit task. Moreover, identification of the letter did not depend on the similarity of the target and distractor colors. The total numbers of letter feature errors were $3.8 \%, 4.0 \%$, and $4.0 \%$ for the identical, similar, and dissimilar trials, respectively $[F(2,22)<1.0]$.
Figure 2 presents the percentages of color feature and conjunction errors. A response was classified as a conjunction error when the subject reported the distractor color as the target color and reported the correct letter. ${ }^{1}$ A response was classified as a color feature error when the subject reported a color that was not part of the display. The independent variable in Figure 2 is the relationship between the target and the reported color.

Subjects were more likely to respond with a color similar to the target color than with a dissimilar color. This effect was obtained for both feature errors $[t(11)=2.55$, $p<.05]$ and conjunction errors $[t(11)=4.66$, $p<.001$ ]. The former effect provides empirical support for our choice of similar and dissimilar colors. More interesting for our present concerns is that the significant effect of similarity on conjunction errors supports the hypothesis that the process of feature integration is constrained by feature similarity.

The analysis of illusory conjunctions must be qualified. The responses labeled as illusory conjunctions in Figure 2 include all trials in which the distractor color is reported as the color of the target letter. We assume that some of these errors represent true illusory conjunctions. However, some of the observed illusory conjunctions are actually feature errors. That is, on trials in which the subjects are unable to identify the target color, they must choose one of the four possible colors, perhaps constraining their choice through the use of partial information from the target. Sometimes the color reported in these situations will be the distractor color and these true feature errors will be recorded as illusory conjunctions. Similarly, the color reported may be the target color, and thus a correct response will be recorded.

Consider the hypothetical example in Table 1 . The feature errors $(5+5)$ are not the total number of times the



Figure 2. Preportion of conjunction and feature errors in Experiment 1 , as a function of the relationship between the correct color and the reported color. 
Table 1

Hypothetical Distribution

\begin{tabular}{|c|c|c|c|c|}
\hline \multirow[b]{2}{*}{$\begin{array}{l}\text { Total } \\
\text { Trials }\end{array}$} & \multirow[b]{2}{*}{$\begin{array}{c}\text { Correct } \\
\text { Responses }\end{array}$} & \multirow[b]{2}{*}{$\begin{array}{l}\text { Conjunction } \\
\text { Errors }\end{array}$} & \multicolumn{2}{|c|}{ Feature Errors } \\
\hline & & & $\begin{array}{c}\text { Nondisplay } \\
\text { Color } 1\end{array}$ & $\begin{array}{c}\text { Nondisplay } \\
\text { Color } 2\end{array}$ \\
\hline 100 & 70 & 20 & 5 & 5 \\
\hline
\end{tabular}

subject did not perceive the color and guessed. Subjects will guess the target color on $25 \%$ of the trials (correct response), the distractor color $25 \%$ of the time (conjunction error), and a nondisplay color on $50 \%$ of the guesses (feature error). Thus, the observed feature errors are only $50 \%$ of the color guesses. If subjects did not make any true illusory conjunctions, there would be five illusory conjunctions by chance alone [i.e., $(5+5) \cdot 0.50$ ]

To obtain an estimate of true illusory conjunctions $\left(I C_{\text {true }}\right)$, we subtract out the portion of the illusory conjunctions ( $\left.I C_{\text {observed }}\right)$ that are the actual feature errors. That is:

$$
\left.I C_{\text {true }}=I C_{\text {observed }}-\text { feature errors } \cdot 0.50\right) .
$$

If there were no true illusory conjunctions, then $I C_{\text {true }}$ would equal zero. This general procedure for treating feature errors that are recorded as illusory conjunctions has been used in numerous illusory conjunction studies (e.g., Cohen \& Ivry, 1989; Prinzmetal \& Millis-Wright, 1984; Treisman \& Schmidt, 1982). ${ }^{2}$

We calculated estimates of true illusory conjunctions for each subject separately for similar and dissimilar trials. From these data, we first determined whether illusory conjunctions were obtained in our paradigm and then evaluated the similarity hypothesis. The percentage of true illusory conjunctions was significantly greater than zero on both similar $[t(11)=5.07, p<.001]$ and dissimilar trials $[t(11)=3.10, p<.01]$. The mean percentages of true illusory conjunctions were $14.6 \%$ and $8.1 \%$ for the similar and dissimilar conditions, respectively. The difference between these two values is significant $[t(11)=4.64$, $p<.001]$. This analysis demonstrates that, in addition to the effect of feature errors, color similarity constrains illusory conjunctions. Subjects are more likely to erroneously conjoin similar colors than dissimilar colors.

Finally, subjects were more accurate overall on identical trials than on similar and dissimilar trials. The percent correct for identical, similar, and dissimilar trials was $92.8 \%, 77.8 \%$, and $83.2 \%$, respectively $[F(2,22)=$ $21.86, p<.001]$. This is consistent with previous results showing that performance can be better with identical distractors than with similar distractors (see, e.g., Shapiro \& Krueger, 1983). However, in the present experiments, the overall comparison of the three conditions is not meaningful, because subjects cannot make an illusory conjunction on identical trials.

\section{EXPERIMENT 2}

Illusory conjunctions require the combination of two features, a color and a form. Color similarity was manipulated in Experiment 1, and the results indicated that illu- sory conjunctions were more likely when the display contained similar colors. Experiment 2 was designed to provide the analogous test for shape similarity. Specifically, would there be more illusory conjunctions between items that were similar in shape than between items that were dissimilar in shape? This second test of feature similarity should provide insight into the generality of feature similarity as a constraint underlying the occurrence of illusory conjunctions.

\section{Method}

Stimuli. Except for the letter set, all of the stimulus materials were unchanged. The same set of stimulus colors was used (red, orange, green, blue), and the sizes and arrangement of the colored letters, alerting stimulus, digits, and mask were identical to those in Experiment 1.

Four different letters were included: W, M, T, and L. Two of the letter pairs, $W / M$ and $T / L$, were classified as similar; all other combinations formed dissimilar pairs (see Townsend, 1971).

The selection of stimuli for each trial was similar to that in Experiment 1 , but with letter and color manipulations reversed. The two colors were selected with the constraint that one color be either red or orange and the other color either green or blue. One color served as the target and the other as the distractor. Unlike in Experiment 1 , in which the target letter was always from one pair of letters, the target color was counterbalanced across subjects in Experiment 2. For half of the subjects, the target color was from the pair red/orange; for the other half, the target color was from the pair green/blue. Two letters were then selected out of all possible letter pairs and assigned to one of the two chosen colors. The conditions identical, similar, and dissimilar refer to the relationship between the target and distractor letters.

Procedure. Sixteen subjects, none of whom had participated in Experiment 1, were recruited as before. On each trial, the subject reported first whether the digits were identical, then the form (letter) of the target color, and, last, the target color. Thus, an example of the responses from a trial for a subject in the red/orange subgroup might be to press the keys corresponding to "match," "W," and "red." Note that the order of report for the colored letters was reversed from that of Experiment 1, so that the crucial information was again reported immediately after the digits.

All other aspects of the procedure were unchanged. The exposure duration of the stimulus display was always $86 \mathrm{msec}$ on the last practice block and adjusted according to the criterion developed for Experiment 1. The adjustment procedure produced a mean exposure time over all experimental blocks of $88.8 \mathrm{msec}$ (range: $57-114 \mathrm{msec}$ ).

\section{Results and Discussion}

Overall performance was similar to that observed in Experiment 1 . The subjects were correct on the digit matching task on $87.4 \%$ of the trials, with the percent correct being similar across conditions $[F(2,30)=1.31$, $p>.25$ ]. Performance on the colored letter task was unaffected by performance on the digit task, so the analyses of the colored letter task include all trials, regardless of performance on the digit task. The total numbers of color feature errors are $2.3 \%, 1.9 \%$, and $2.0 \%$ for the identical, similar, and dissimilar conditions. Identification of the color did not depend on the similarity of the target and distractor letters $[F(2,30)<1.0]$.

Figure 3 presents the percentages of conjunction and feature errors for the similar and dissimilar trials. In this experiment, conjunction and feature errors refer to incor- 


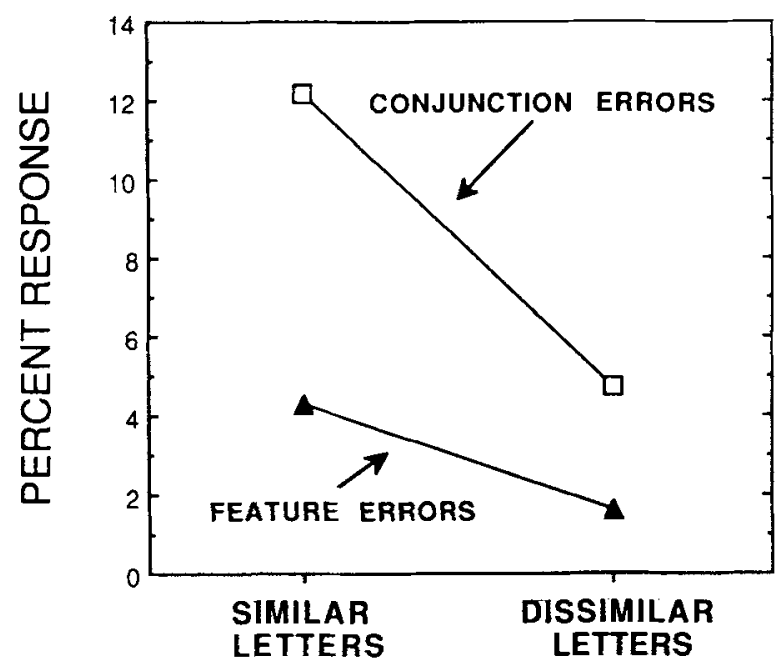

Figure 3. Proportion of conjunction and feature errors in Experiment 2, as a function of the relationship between the correct letter and the reported letter.

rect reports of the target letter: A conjunction error was recorded when the subject reported the letter in the distractor color as the target; a feature error was recorded when the subject reported a letter that was not included in the display. The independent variable in Figure 3 is the relationship between the target and the reported letter.

As in Experiment 1, a similarity effect was obtained for both types of errors. The effect at the feature level $[t(15)=4.44, p<.001]$ verifies our selection of similar and dissimilar letter pairs. The effect at the conjunction level $[t(15)=4.86, p<.001]$ demonstrates that erroneous conjunctions are more likely to occur when the display contains similar letters. We estimated true illusory conjunctions from the observed percentages, as in Experiment 1. As before, this estimation procedure was applied separately for the similar and dissimilar conditions, yielding estimates of true illusory conjunctions of $10.0 \%$ for the similar trials and $3.9 \%$ for the dissimilar trials. Both of these values are significantly greater than zero [similar: $t(15)=4.42, p<.001$; dissimilar: $t(15)$ $=3.38, p<.01]$, indicating that the illusory conjunctions cannot be attributed to guessing. Moreover, the percentage of true illusory conjunctions for similar trials is significantly different from that obtained on dissimilar trials $[t(15)=3.89, p<.001]$. Even after the effect of similarity on feature errors is subtracted, illusory percepts are more likely to occur when the target and distractor are similar in shape.

\section{EXPERIMENT 3}

The first two experiments demonstrated that feature similarity should be considered an additional factor constraining the occurrence of illusory conjunctions. The effect was observed with manipulations of both color and shape. However, in each experiment, the effect of similarity was also found for feature errors. Not only were subjects more likely to report an illusory conjunction with similar stimulus pairs, but, when reporting items that were not part of the display, they were more likely to report a feature that was similar to the target. To overcome the problem presented by this two-pronged effect, we subtracted the proportion of feature errors expected by chance from the observed conjunction errors. Similarity still affected illusory conjunctions.

We took a different tack in the next two experiments, following an analytic strategy developed by Prinzmetal, Presti, and Posner (1986, Experiment 3). Pursuing a different question, Prinzmetal, Presti, and Posner encountered the same problem we had: how to separate the effect of a variable on processing individual features and conjunctions of features. To overcome this, they simply modified their experimental method in order to minimize the occurrence of feature errors. Since conjoining features is a more demanding task (see, e.g., Treisman \& Gelade, 1980), they expected that illusory conjunctions would still be observed even when feature errors were reduced to a minimum. Indeed, this is what they found.

We utilized the same strategy, reducing the effect of feature errors in three ways. First, the digit task was eliminated. Second, we changed our criterion for adjusting exposure duration, in order to make the task easier. Third, we increased the number of items in the display that contained the relevant features, thus increasing the likelihood that the features presented would be perceived. For the color manipulation of Experiment 3, each trial contained five colored letters, three in one color and two in another. For the letter manipulation of Experiment 4, five colored letters were also presented, three of one shape and two of another. These modifications were expected to reduce the number of color feature errors in Experiment 3 and letter feature errors in Experiment 4 (see also Prinzmetal, Presti, \& Posner, 1986). We can still assess whether the similarity effect persisted for the remaining illusory conjunctions.

\section{Method}

Stimuli. The stimuli were drawn from the letter set $\mathrm{X}, \mathrm{T}, \mathrm{C}$, and $S$ and the color set red, orange, green, and blue. The letter $C$ was substituted for the letter $O$, because it was deemed more similar to $\mathbf{S}$. The dimensions of each letter and the color settings were the same as in Experiment 1.

Five letters, two colors, and the locations for the stimuli were determined before each trial. The group of letters consisted of a single target letter, either an $\mathrm{X}$ or a $\mathrm{T}$, plus two of each distractor letter, $\mathbf{C}$ and $\mathrm{S}$. The letters were arranged into a string of five letters. Two of the letters were assigned one color and three were assigned the other color. This designation was made with the constraint that the first two (or three) letters in the string were of the same color and the last three (or two) letters in the string were of the other color.

There were three types of stimulus arrangements (Figure 4). For Display Type 1, the target was in the central position and had the same color as two of the other letters, one $\mathrm{C}$ and one S. For Display Types 2 and 3, the target was either in the second position from the left or the second position from the right, and it was al- 
STIMULUS

TYPE EXAMPLE

CONJUNCTION

23.67

5.00

$10 \mathrm{CSSC}$

27.83

4.33

2

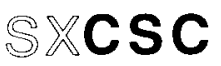

3

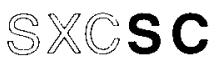

15.92

5.42

\section{TARGET LETTER : $X$}

Figure 4. Sample stimuli of the three types of stimulus arrangements in Experiment 3, with mean number of conjunction and feature errors per subject. Feature errors include reports of colors and letters not included in the display.

ways bordered by one C and one S. For Display Type 2, the target was flanked by two different colors; the neighboring distractor on the lateral edge of the five-letter string was assigned the same color as the target. For Display Type 3, the target was part of a substring of three letters, all in the same color. Thus, in Display Types 1 and 2, the target was flanked by different colors, whereas in Display Type 3, the target was surrounded by letters in the same color. The group of letters containing the target was randomly set to be on either the left or the right side of the stimulus string.

The three types of displays were designed to prevent the subjects from focusing on the middle letter and simply reporting the color that was used for three of the five letters. This strategy is negated in two of the display types, since the target is displaced one position from the center of the string. Moreover, to report the most common color would produce an incorrect response for Display 2 , since there were only two letters in the target color. Last, comparison of Displays 1 and 2 with Display 3 allowed us to see whether a distance effect would be obtained with this paradigm, since an illusory conjunction with Display 3 would require one of the features to migrate across an intervening letter. The three display types were presented equally often.

The stimulus string was randomly positioned at one of four locations. At a viewing distance of $100 \mathrm{~cm}$, the outermost horizontal edge for all positions was displaced $2.35^{\circ}$ to the left or right of the center of the monitor. The stimulus string was also displaced $1.07^{\circ}$ above or below the horizontal meridian. As before, each letter was $0.43^{\circ}$ wide. However, the edge-to-edge distance was reduced by one pixel, to a distance of $0.09^{\circ}$. Thus, the entire stimulus string was $2.51^{\circ}$ wide. The distance between the two horizontal bars spanned $6.50^{\circ}$. A colored pattern mask, measuring $5.21^{\circ} \times 5.35^{\circ}$, was used to terminate the stimulus display.

Procedure. Each trial began with the presentation of the horizontal bars. After $500 \mathrm{msec}$, the colored letter string was added to the display. After a variable period of time, the stimulus display was replaced by the colored pattern mask for $200 \mathrm{msec}$. The subject entered two responses, first the color of the target letter and then the identity of the target letter. Feedback was provided only when subjects reported a color or letter absent from the display. The intertrial interval was $200 \mathrm{msec}$. The interval from the onset of the alerting stimulus to the onset of the colored letter display as well as the ITI was reduced in order to reduce the time per trial. This allowed us to collect more data in a 1-h session.

An experimental session was composed of five practice blocks of 20 trials each and four experimental blocks of 96 trials each. The exposure durations for the first four practice blocks were 229 ,
143,114 , and $86 \mathrm{msec}$, respectively. The exposure duration for the final practice block was set according to the following criteria. If the subject was correct on at least 39 of the $\mathbf{4 0}$ possible responses ( 20 trials/block with 2 responses/trial), the exposure duration was reduced by 1 vertical synch cycle (approximately $14.28 \mathrm{msec}$ ). If the subject was correct on less than 37 responses, the exposure duration was increased by 1 vertical synch cycle. Otherwise the exposure duration was held constant. The exposure duration for the first test block was determined in a similar manner, on the basis of the subjects' performance in the final practice block. For subsequent test blocks, the exposure duration was reduced if the subject was correct on 190 out of 192 color and letter responses (96 trials/block) and increased if the subject was correct on less than 182 responses. This strict criterion was adopted to reduce the number of feature errors. The adjustment procedure produced a mean exposure time over all experimental blocks of $76.8 \mathrm{msec}$ (range: 43-114 msec).

As in Experiment 1, there were three types of trials, depending on the color relation between the subgroup of letters containing the target and the subgroup of letters containing only distractors: identical, similar, and dissimilar. Note that in the identical condition, all five letters were the same color. All possible combinations of color pairs were presented equally often over a block of experimental trials, and equally often under each of the three display types. Thus, there were nine primary conditions ( 3 display types $\times 3$ color relations). Each of the 16 color combinations was presented twice per block or eight times over the four experimental blocks.

Thirteen naive subjects were recruited. The data for 1 subject were eliminated. This subject made over twice as many color feature errors as all but 1 of the other subjects and made twice as many total feature errors as the remaining subject.

\section{Results and Discussion}

The total numbers of letter feature errors were $5.2 \%$, $5.7 \%$, and $6.3 \%$ for the identical, similar, and dissimilar conditions, respectively. These percentages are slightly higher than had been observed in Experiment 1, which may reflect greater lateral masking of the letter identity. The target was always surrounded on two sides by distractor letters in the current experiment. Nonetheless, there was no difference between conditions in terms of performance on the letter identification task $[F(2,22)<1.0]$.

The modifications introduced in Experiment 3 were successful in reducing color feature errors. The overall total was under $1 \%$. As shown in Figure 5, similar colors produced more conjunction errors $[t(11)=2.58$, $p<.05]$ and more feature errors $[t(11)=1.99$, $p<.05]$. We estimated true illusory conjunctions as in the previous experiments. Analyses of these data revealed that the percentage of true illusory conjunctions was significantly greater than zero for both similar $[t(11)=$ $16.39, p<.001]$ and dissimilar $[t(11)=13.18$, $p<.001]$ trials. There were $25.6 \%$ true illusory conjunctions on similar trials and $22.1 \%$ on dissimilar trials. Subjects were more likely to make a true illusory conjunction when the target and distractor colors were simi$\operatorname{lar}[t(11)=2.47, p<.05]$.

In sum, these data are again supportive of the similarity constraint. While we were able to nearly eliminate color feature errors, the remaining errors did tend to indicate a similarity effect at the feature level. Nonetheless, one informal analysis is further supportive of our claim 


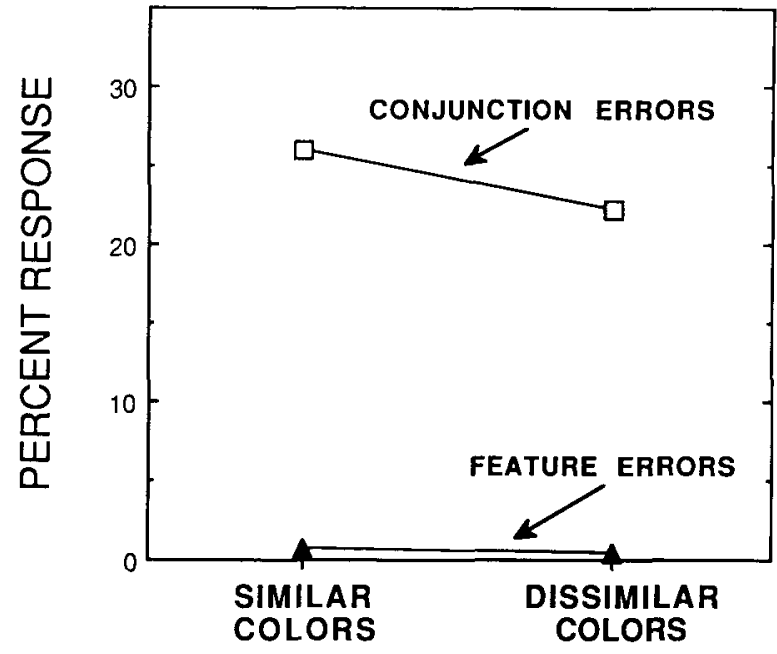

Figure 5. Proportion of conjunction and feature errors in Experiment 3 , as a function of the relationship between the correct color and the reported color.

that the similarity constraint is evident for conjoining features. Seven subjects committed less than $1 \%$ color feature errors on trials with similar colors, and 5 committed more than $1 \%$. The similarity effect was essentially identical for both subgroups, averaging about $4 \%$ more illusory conjunctions when the display contained similar colors $(4.0 \%$ and $3.5 \%$ for the subjects who committed less than and greater than $1 \%$, respectively). In contrast, the mean number of color feature errors was greater for similar colors only for the 5 subjects who made more than $1 \%$ color feature errors. We are, of course, not arguing that similarity does not influence feature errors. Rather, when feature errors are virtually eliminated, an effect on illusory conjunctions remains. Thus the effect on illusory conjunctions cannot be due solely to misclassifying some feature errors as conjunction errors.

One remaining aspect of the similarity effect should be noted. In Experiment 1, we had found that displays with similar colors had produced almost twice as many illusory conjunctions as had displays with dissimilar colors. In the current experiment, the percentage increase was only $16 \%$. It is possible that, on some trials, the subject did not clearly perceive the color of the target. However, the other letters in the display provided information as to which colors were present. If the subject randomly responded with one of these colors, the subject would be correct on some trials and produce illusory conjunctions on others. Since errors of this type could occur for both similar and dissimilar trials, the similarity effect would be diluted.

As noted above, the three different display types provided a number of controls that eliminated certain potential response strategies. However, a number of interesting questions can be asked by examining the error data as a function of display type. We examined these effects by entering the number of conjunction errors and the combined color and letter feature error total into a 3 (display type) $\times 2$ (error type) repeated measures analysis. Both main effects were significant [display type: $F(2,22)=14.45, p<.001$; error: $F(1,11)=112.01$, $p<.001]$, as was the interaction $[F(2,22)=21.54$, $p<.001]$. Post hoc analyses were made with Newman-Keuls tests. The different display types had no effect on the feature errors (Figure 4). Subjects were equally likely to report a color or letter not present in the display, regardless of the position of the target or the color of the surrounding letters.

In contrast, all pairwise comparisons obtained significance for the conjunction errors. Identical results were obtained using the estimates of true illusory conjunctions, in which case the mean proportions were 21.17, 25.67, and 13.21 for Types 1, 2, and 3, respectively. Consider first the finding that Display Type 3 yielded fewer conjunction errors than did either of the other displays. This indicates that illusory conjunctions are more likely between adjacent items. However, the comparison of Display Types 1 and 3 is confounded with the position of the target, whereas the comparison of Display Types 2 and 3 involves a confound of the number of distractor colors. Nonetheless, the fact that both comparisons were significant could be interpreted as a distance effect.

Second, the greatest number of illusory conjunctions was observed with Display Type 2 . This was the only display that contained two letters in the target color and three distractor letters in the other color. A tentative implication of these results is that illusory conjunctions are constrained by the number of items containing a given alternative feature, rather than just the presence of that feature. This is reasonable, in that each additional item in the distractor color will provide an opportunity for an illusory conjunction. Alternatively, there may be a bias to report the center color, which would only yield illusory conjunctions in Display Type 2.

\section{EXPERIMENT 4}

In Experiment 4, letter similarity was tested with the methodology of Experiment 3. Again, our goal was to see whether or not a similarity constraint would be observed when feature errors were minimized.

\section{Method}

Stimuli. The stimulus sets were the same as in Experiment 2. The letters were drawn from the set T, L, M, and W, and the color set consisted of red, orange, green, and blue.

The displays were essentially identical to those used in Experiment 3, with the exception that the roles of the letter and color manipulations were reversed. A string of five colored letters was selected for each trial, composed of a target subgroup and a distractor subgroup (Figure 6). One letter was used for all members of the target subgroup, and a second letter was used for the distractor subgroup. For half the subjects, the target color was red or orange, and the distractors were blue and green. The task for these subjects was to report the identity of the letter in the target color and then specify the color of that letter; for the other half, the target color was blue or green and the distractors were red and 


\begin{tabular}{|c|c|c|c|}
\hline \multicolumn{2}{|c|}{ STIMULUS } & \multicolumn{2}{|c|}{ PERCENT OBSERVED } \\
\hline TYPE & EXAMPLE & CONJUNCTION & FEATUR \\
\hline 1 & & 15.00 & 5.50 \\
\hline 2 & & 15.07 & 5.36 \\
\hline 3 & 《] & 5.14 & 4.86 \\
\hline
\end{tabular}

Figure 6. Sample stimuli of the three types of stimulus arrangements in Experiment 4, with mean number of conjunction and feature errors per subject. Feature errors include reports of colors and letters not included in the display.

orange. The four nontarget letters were split among the remaining two distractor colors. For example, if the target was red, two of the-distractors were green and two were blue. As in all of the experiments, all combinations of the four letters were equally possible for a given trial.

Procedure. Fourteen naive subjects were recruited. The procedure was identical to that of Experiment 3, but with two exceptions. Subjects were required to report the identity of the target letter first and then the color of the target letter. Also, on the basis of a pilot study, the exposure durations for Practice Blocks 2-4 were increased by 1 vertical synch to 157,129 , and $100 \mathrm{msec}$, respectively. The exposure duration for the last practice block and experimental blocks were set according to the criterion used in Experiment 3 . The mean exposure duration was $118.4 \mathrm{msec}$ (range: 100-171 msec). These times were longer than those used in any of the other experiments.

\section{Results and Discussion}

There were no consistent differences in the total numbers of color feature errors for the three conditions, which averaged $2.5 \%, 2.9 \%$, and $3.6 \%$ for the identical, similar, and dissimilar conditions, respectively $[F(2,26)=$ $1.48, p>.25]$. These values are slightly higher than those in Experiment 2, perhaps reflecting lateral masking. Note that the exposure durations were longer and that there was no digit task, two factors which should have led to improved performance.

The mean average of letter feature errors was $2 \%$. Although this represents over a $60 \%$ reduction from Experiment 2 , we did not succeed in achieving the level of accuracy obtained in Experiment $3{ }^{3}$ Subjects made more feature and conjunction errors when the display contained similar letters than when the display contained dissimilar letters (Figure 7). This effect was obtained for conjunction errors $[t(13)=4.50, p<.01]$ and feature errors $[t(13)=7.23, p<.01]$. An analysis of the true illusory conjunctions revealed that the probability of these reports was significantly greater than zero for similar $[t(13)=$ $11.88, p<.001]$ and dissimilar $[t(13)=7.51$, $p<.001]$ trials. Most important was that more true il- lusory conjunctions were observed with displays containing similar letters rather than dissimilar letters $[18.8 \%$ vs. $8.3 \%, t(13)=6.38, p<.001$ ]

The results demonstrate again that illusory conjunctions are affected by similarity in shape. We were unable to eliminate the effect at the feature level. Nonetheless, we were able to obtain a robust effect for the conjunction error data. The subjects made illusory conjunctions on almost $20 \%$ of the trials for displays containing similar letters, even though they were able to report the target letter on $98 \%$ of the trials.

Comparison of the different display types presented a pattern similar to that observed in Experiment 3. There was an interaction between the type of error made and the different displays $[F(2,26)=21.26, p<.001]$. As shown in Figure 6, there was no difference between the three display types in terms of feature errors. Post hoc analyses with Newman-Keuls tests revealed that fewer conjunction errors were obtained with Display Type 3 than with either of the other two displays. With this display, the target letter was surrounded by two identical letters, rather than being flanked by one identical and one different letter. These results are again indicative of a distance effect. Illusory conjunctions were much more likely to occur between adjacent features than between features separated by an intervening item. Identical results were obtained with true conjunction errors. The estimated true conjunction errors were $12.25 \%, 12.39 \%$, and $2.71 \%$ for Types 1, 2, and 3, respectively.

Unlike in Experiment 3, Display Types 1 and 2 produced an equal number of illusory conjunctions. Subjects were equally likely to report an illusory conjunction when the display contained two or three tokens of the distractor item. Whereas Experiment 3 involved an additional distractor color, Experiment 4 involved an additional distractor letter. This difference is in agreement



Figure 7. Proportion of conjunction and feature errors in Experiment 4 , as a function of the relationship between the correct letter and the reported letter. 
with Kanwisher (1989), who found greater "repetition blindness" for letters in comparison to colors. Subjects were more likely to miss a repeated letter than they were to miss a repeated color.

\section{GENERAL DISCUSSION}

The four experiments reported in this paper consistently demonstrated that illusory conjunctions are more likely to occur between items composed of similar features than between items composed of dissimilar features. This effect was obtained for manipulations of both color similarity (Experiments 1 and 3) and letter similarity (Experiments 2 and 4). These results require that feature similarity be added to the list of constraints underlying the phenomena of illusory conjunctions. Contrary to the original formulation of Treisman's feature integration theory (Treisman \& Gelade, 1980; Treisman \& Schmidt, 1982), features are not free floating, but subject to a rich variety of constraints that reflect spatial, attentional, and featural properties, as well as grouping, of the stimulus display.

The current results appear to contradict the conclusions of Treisman and Schmidt (1982) regarding similarity. These researchers failed to find an effect of similarity in their seminal paper on illusory conjunctions (Experiment 4). There are a number of methodological differences between the two experiments, including the task and interitem spacing. Moreover, their operational definition of similarity is quite different from that employed in the current research. They assessed whether illusory conjunctions were more likely to occur between stimuli that were more similar across dimensions. For example, if the two items were of the same size and shape, would they be more likely to exchange color? In contrast, we examined the effect of similarity within a dimension. Will items in similar colors be more likely to exchange color? Thus, the similarity constraint may be limited to intradimensional manipulations and not reflect any sort of similarity metric in multidimensional space.

These experiments further validate the usefulness of the illusory conjunction paradigm. First, the constraints revealed in the study of illusory conjunctions provide a rich source of information on how the visual system combines features during object perception. Second, the similarity effect convincingly demonstrates that illusory conjunctions are a genuine perceptual phenomenon, and not just the result of a propensity to guess the color of another display item. Consider the situation when a subject perceives the two colors and the target letter in displays such as those used in our experiments. If the target letter is not linked to one of the colors, the subject should simply guess one of the two colors. On half the trials, the subject would be correct; for the remaining half, an illusory conjunction would be recorded. Indeed, it is possible that many illusory conjunctions occur in this manner. However, there is no reason for perceptual similarity to affect guessing in a manner consistent with the current results. For example, it is reasonable to suppose that if illusory con- junctions are the result of guessing between two perceived colors, then this should occur equally often for similar and dissimilar pairs. Moreover, we expect that any bias would work against a similarity effect. When subjects are given one color name and asked to generate another color name in a free association task, their responses are not dominated by similar color names. For example, if a subject is given red, the most typical response is "blue," not "orange" or "yellow" (Postman \& Keppel, 1970).

The issue of similarity has recently been raised in the study of visual search. Wolfe, Cave, and Franzel (1989) have argued that visual search involves an essentially parallel process in which candidate features are competing for focal attention. According to this model, similarity increases the competition by increasing the number of viable candidates. In a more general application of this idea, Duncan and Humphreys (1989) have argued that feature detection reflects two distinct similarity relations: first, the similarity between the target(s) and distractors, and second, the similarity between different distractors. Both of these papers focus on processes involved in the detection/identification of features and postulate that similarity will make it harder to detect either a target defined by a single feature or conjunctions composed of these features. In other words, similarity reduces discriminability.

This argument, however, does not account for the effect of similarity on illusory conjunctions. For example, in Experiment 3, the colors were accurately identified (and thus discriminated), yet illusory conjunctions were still more likely to occur between similar colors than they were to occur between dissimilar colors. These data suggest that there is an additional effect of similarity at the processing stage in which illusory conjunctions are formed. Thus, although a similarity principle may offer a unified account of feature and conjunction visual search, such parsimony may not be realized between visual search and illusory conjunctions (see also Duncan \& Humphreys, 1989).

To this point, we have presented what the similarity effect does not show. It is not the result of guessing and it cannot be attributed to confusion in feature identification. There are a number of possible interpretations for the effect; we will discuss three. First, as suggested in the preceding paragraph, there may be different effects of similarity in terms of identifying features and conjoining features. The effect of similarity could be modeled as an aperture over a region in a dimensional space. Identification can be thought of as activation of a region on the dimensional surface passing through the aperture. Since similarity is represented in the dimensional space as the distance between neighboring points, it would follow that items similar to a target would pass through the aperture more often than would dissimilar items. The additional effect of similarity on illusory conjunctions would require that the information used for conjoining features not be identical to that used for identifying features. That is, the information must pass through separate apertures, one that 
yields the similarity effect on feature identification and one that yields an additional similarity effect for feature integration. According to this model, similarity effects could reflect perceptual confusions or decision effects.

A second possibility is that the similarity effect on illusory conjunctions is at least partially distinct from the similarity effect on detection processes in visual search. As noted above, the model of Wolfe et al. (1989) would attribute the latter effect to a competition between stimuli for a focal attention process. Inputs lead to increasing activation of their features, and, over time, discrimination becomes easier. The effect of similarity on illusory conjunctions might reflect the opposite process. Assume that the features have been identified prior to a conjoining stage of processing. Perceptual information of these features may then begin to decay, leading to an increase in confusion, which is especially marked for similar features. That is, feature representation may be a continuous, nonmonotonic process in which activation accrues and then dissipates. Errors or interference effects that occur during the increase in activation will reflect a similarity effect in feature identification, whereas illusory conjunctions occur during the decrease in activation, reflecting a second similarity effect due to information loss. ${ }^{4}$

Third, the similarity effect may reflect a derivative manifestation of the grouping effects observed in previous studies of constraints underlying the formation of illusory conjunctions (Prinzmetal \& Keysar, 1989). The more similar two items are in some attribute (e.g., color), the more likely it is that they will form a perceptual group. Since illusory conjunctions are more likely to occur within perceptual groups rather than between perceptual groups (Prinzmetal, 1981; Prinzmetal \& Keysar, 1989), the similarity effect may result from grouping tendencies between similar letters or colors. One test of this hypothesis would be to employ displays that contain a pair of distractors, one similar and one dissimilar to the target, and to manipulate the grouping relations of the three items. For example, grouping could be manipulated spatially by locating the target adjacent to the similar distractor or to the dissimilar distractor. We would predict an interaction between similarity and distance. The effect of similarity would be evident in the analysis of illusory conjunctions between adjacent items, but eliminated (or reduced) for illusory conjunctions between items that are not part of the same perceptual group. Grouping could, of course, be manipulated in other ways.

Perceptual grouping could be considered part of the more general claim that illusory conjunctions are primarily due to poor location tagging of features. As found in this and in other studies (e.g., Cohen \& Ivry, 1989; Wolford \& Shum, 1980), illusory conjunctions are more likely between items that are close together. Perceptual grouping affects illusory conjunctions because items that form a perceptual group are closer in psychological space (Coren \& Girgus, 1980). The claim is that psychological distance, rather than physical distance, is critical for illusory con- junctions. Any factor that affects psychological space would affect illusory conjunctions. Cohen and Ivry's (1989) finding that the pattern of illusory conjunctions is affected by the distribution of spatial attention can be viewed in this manner. Assuming that attended items are distinct from unattended items (i.e., closer in psychological space), there should be more illusory conjunctions between attended items. These ideas provide a framework to account for why illusory conjunctions will occur between items within the focus of attention or between items outside the focus of attention, but not across the focus of attention (Cohen \& Ivry, 1989; Treisman \& Schmidt, 1982).

\section{REFERENCES}

COHEN, A., \& IVRy, R. (1989). Illusory conjunctions inside and outside the focus of attention. Journal of Experimental Psychology: Human Perception \& Performance, 15, 650-663.

Coren, S, . \& Girgus, J. S. (1980). Principles of perceptual organization and spatial distortion: The Gestalt illusions. Joumal of Experimental Psychology: Human Perception \& Performance, 6, 404-412.

Duncan, J., Humphreys, G. W. (1989). Visual search and stimulus similariry. Psychological Review, 96, 433-458.

Gallant, J. L., \&arner, W. R. (1988). Some effects of distance and structure on conjunction errors. Bulletin of the Psychonomic Society, 26, 323-326.

InDow, T. (1988). Multidimensional studies of Munsell color solid. Psychological Review, 95, 456-470.

KANWISHER, N. G. (1989, November). Types and tokens in visual information processing. Paper presented at the 30th Annual Meeting of the Psychonomic Society, Atlanta.

Kfele, S., Cohen, A., Ivry, R., Liotti, M., YeE, P. (1988). Tests of a temporal theory of attentional binding. Journal of Experimental Psychology: Human Perception \& Performance, 14, 444-452.

LeVine, D., Warach, J., FARAH, M. (1985). Two visual systems in mental imagery: Dissociation of "what" and "where" in imagery disorders due to bilateral posterior cerebral lesions. Neurology, 35 , 1010-1018.

MClean, J. P., Broadbent, D. E., \& Broadbent, M. H. (1982). Combining attributes in rapid serial visual presentation tasks. Quarterly Joumal of Experimental Psychology, 35A, 171-186.

MunSEl. Color Company. (1929). Munsell book of color. Baltimore: Author.

Newcombe, F., Ratcliffe, G., Damasio, H. (1987). Dissociable visual and spatial impaiments following right posterior lesions: Clinical, neuropsychological, and anatomical evidence. Neuropsychologia, 25, 149-161

Postman, L., KePPEL, G. (1970). Norms of word association. New York: Academic Press.

Prinzmetal, W. (1981). Principles of feature integration in visual perception. Perception \& Psychophysics, 30, 330-340.

Prinzmetal, W. (1990). Neon colors illuminate reading units. Journal of Experimental Psychology: Human Perception \& Performance, 16, 584-597.

Prinzmetal, W., \& Keysar, B. (1989). Functional theory of illusory conjunctions and neon colors. Journal of Experimental Psychology: General, 118, 165-190.

Prinzmetal, W., \& Miclus-Wrught, M. M. (1984). Cognitive and linguistic factors affect visual feature integration. Cognitive Psychology, 16, 305-340.

Prinzmetal, W., Presti, D., Posner, M. (1986). Does attention affect feature integration? Joumal of Experimental Psychology: $\mathrm{Hu}$ man Perception \& Performance, 12, 361-369.

Prinzmetal, W., Treiman, R., RHo, S. (1986). How to see a reading unit. Journal of Memory \& Language, 25, 461-475. 
SEIDENBERG, M. (1987). Sublexical structures in visual word recognition: Access units or orthographic redundancy? In M. Coltheart (Ed.), Attention and performance XII (pp. 245-263). Hillsdale, NJ: Erlbaum.

ShAPIRo, R. G., \& KRUEGER, L.E. (1983). Effect of similarity of surround on target-letter processing. Journal of Experimental Psychology: Human Perception \& Performance, 9, 547-559.

SHEPARD, R. N. (1980). Multidimensional scaling, tree-fitting, and clustering. Science, 210, 390-398.

TOWNSEND, J. T. (1971). Theoretical analysis of an alphabetic confusion matrix. Perception \& Psychophysics, 9, 40-50.

Treisman, A., \& Gelade, G. (1980). A feature-integration theory of attention. Cognitive Psychology, 12, 97-136.

TrFisman, A., SCHMid, H. (1982). Illusory conjunctions in the perception of objects. Cognitive Psychology, 14, 107-141.

UNGERLEIDER, L. G., \& MishKIN, M. (1982). Two cortical visual systems. In D. J. Ingle, M. A. Goodale, \& R. J. W. Mansfield (Eds.), Analysis of visual behavior (pp. 549-586). Cambridge, MA: MTT Press.

Wolfe, J. M., CAVE, K. R., \& Franzel, S. L. (1989). Guided search: An alternative to the feature integration model for visual search. Journal of Experimental Psychology: Human Perception \& Performance, $15,419-433$

Wolford, G. (1975). Perturbation model for letter identification. Psychological Review, 82, 184-199.

WolfoRD, G. SHUM, K. H. (1980). Evidence for feature perturbations. Perception \& Psychophysics, 27, 409-420.

\section{NOTES}

1. It is possible to argue that trials on which the subject reported the distractor color and the incorrect letter should also be considered illusory conjunctions. However, these occurred infrequently, and inclusion of them does not change any of the statistical analyses.

2. This estimation procedure is only valid when the distractor color does not provide any information about the target color. Previous studies have generally not made the probability of the target color independent of the distractor color.

3. The same criterion was used in Experiments 3 and 4 to adjust the exposure duration. In both experiments, most errors occurred in the identification of the target letter. However, in Experiment 3, this source of error was of secondary interest, whereas in Experiment 4, the same error was of primary interest. We expect in principle that we could have obtained illusory conjunctions while making the letter identification task relatively easier in comparison with identifying the colors-for example, by using larger letters. However, this would have introduced a large change in many of the details of the stimulus displays, and thus we decided to accept a $2 \%$ letter feature error rate.

4. Jeremy Wolfe suggested the idea of a decay process.

(Manuscript received April 16, 1990; revision accepted for publication August 27, 1990.) 